J. Austral. Math. Soc. 23 (Series A) (1977), 105-111.

\title{
ON CERTAIN SUBALGEBRAS OF A DUAL $B^{*}$-ALGEBRA
}

PAK-KEN WONG

(Received 25 September 1975)

\begin{abstract}
Let $A$ be a dual $B^{*}$-algebra and $A_{p}$ the $p$-class in $A$. We show that the conjugate space of $A_{1}$ is $A^{* *}$, the second conjugate space of $A$. We also obtain a three lines theorem for $A_{p}(1 \leqq p \leqq x)$.
\end{abstract}

\section{Introduction}

Let $H$ be a (complex) Hilbert space, $L C(H)$ the algebra of all compact operators on $H$ and $L(H)$ the algebra of all continuous linear operators on $H$. Then $L C(H)$ is a simple dual $B^{*}$-algebra and every simple dual $B^{*}$-algebra is of this form. Also the second conjugate space of $L C(H)$ is $L(H)$. The class $C_{p}$ $(0<p \leqq \infty)$ of compact operators in $L C(H)$ has many interesting properties and has been studied in various articles (e.g., see Gohberg and Krein (1969) and McCarthy (1967)). In Wong (1974), a similar class of spaces $A_{p}(0<p \leqq$ $x)$ in an arbitrary dual $B^{*}$-algebra $A$ was introduced and studied. The purpose of this paper is to establish three more results for $A_{p}$.

It is well known that the conjugate space of $C_{1}$ is $L(H)$ (see Schatten (1960), p. 47, Theorem 2). In this paper, we generalize this result to an arbitrary dual $B^{*}$-algebra $A$. In fact, we show that the conjugate space of $A_{1}$ is $A^{* *}$, the second conjugate space of $A$. By identifying $A_{1}$ as a subspace of $A^{* * *}$, we prove that for every $F$ in $A^{* * *}, F=G+H$ uniquely with $G \in A_{1}$, $H \in A^{\perp}$ and $\|F\|=\|G\|+\|H\|$. For the case $A$ is a simple algebra, this result was proved by Dixmier (see Schatten (1960), Theorem 5). We also obtain a three lines theorem for $A_{p}(1 \leqq p \leqq \infty)$ which is a generalization of Gohberg and Krein (1969), Theorem 13.1.

\section{Notation and preliminaries}

In this paper, all algebras and linear spaces under consideration are over the field of complex numbers. Definitions not explicitly given are taken from Rickart's book (Rickart (1960)). 
Notation 1. If $B$ is a $B$ anach space, then $B^{*}$ and $B^{* *}$ will be its conjugate space and second conjugate space respectively. Also $B^{* * *}$ will be the conjugate space of $B^{* *}$.

Notation 2. In this paper, $A$ will denote a dual $B^{*}$-algebra with norm $\|\cdot\|$.

REMARK. It is well known that $A^{* *}$ is a $W^{*}$-algebra containing $A$ as a *subalgebra (see Sakai (1971), Theorem 1.17.2). Since $A$ is a dual algebra, by Wong (1973), Theorem 3.1, $A$ is a two-sided ideal of $A^{* *}$.

Let $b$ be a nonzero normal element in $A$ and $\left\{e_{\tau}\right\}$ a maximal orthogonal family of hermitian minimal idempotents in $A$ such that $e_{\tau} b=b e_{\tau}=k_{\tau} e_{\tau}$, where $k_{\tau}$ is a constant. Then it is shown in Wong (1974), that the set $\left\{k_{n}\right\}=$ $\left\{k_{r}: k_{r} \neq 0\right\}$ is countable and independent of the choice of $\left\{e_{r}\right\}$ and

$$
b=\sum_{\tau} e_{\tau} b=\sum_{n} k_{n} e_{n}
$$

where $e_{n} \in\left\{e_{\tau}\right\}$ with $e_{n} b=k_{n} e_{n}$. (2.1) is called a spectal representation of $b$.

Now suppose $A$ is a nonzero element in $A$ and $a^{*} a=\Sigma_{n} r_{n} e_{n}$ is a spectral representation of $a^{*} a$. Since $a^{*} a$ is a positive element, $r_{n}>0$. Put $k_{n}=\sqrt{r_{n}}$ and define

$$
|a|_{p}=\left(\sum_{n} k_{n}^{p}\right)^{1 / p} \quad(0<p<\infty)
$$

and

$$
|a|_{x}=\max \left\{k_{n}: n=1,2, \cdots\right\}
$$

For $a=0$, we define $|a|_{p}=0(0<p \leqq \infty)$. Let

$$
A_{p}=\left\{a \in A:|a|_{p}<\infty\right\} \quad(0<p \leqq \infty) .
$$

It was shown in Wong (1974) that for $1 \leqq p \leqq \propto, A_{p}$ is a dual $A^{*}$-algebra which is a dense two-sided ideal of $A, A=A_{x}$ and $A_{1}=\left\{a b: a, b \in A_{2}\right\}$. Let $b, c \in A_{2}$ and $\left\{f_{r}\right\}$ a maximal orthogonal family of hermitian minimal idempotents in $A$. Then $f_{\tau} c^{*} b f_{\tau}=m_{\tau} f_{\tau}$ for some constant $m_{\tau}$. By Wong (1974), Theorem 4.1, $\sum_{\tau} m_{\tau}$ is absolutely summable and independent of $\left\{f_{\tau}\right\}$ and $A_{2}$ is a proper $H^{*}$-algebra with the inner product defined by $(b, c)=\Sigma_{\tau} m_{\tau}$. For each $a \in A_{1}$, let $a=c^{*} b$ with $b, c \in A_{2}$. Define

$$
\operatorname{tr}(a)=(b, c)=\sum_{\tau} m_{\tau}
$$

Then by Wong (1974), Lemma 4.4, $\operatorname{tr}(a)=\Sigma_{\tau}\left(a f_{\tau}, f_{\tau}\right)$ and $|\operatorname{tr}(a)| \leqq|a|_{1}$. 


\section{The conjugate spaces of $A_{1}$ and $A^{* *}$}

A bounded linear operator $T$ on $A$ is called a right centralizer if $T(a b)=$ $(T(a)) b$ for all $a, b$ in $A$. The set of all right centralizers on $A$ is denoted by $R(A)$.

Lemma 3.1. As normed linear spaces, $A^{* *}$ and $R(A)$ are isometrically isomorphic.

Proof. Let $\left\{x_{\tau}\right\}$ be an approximate identity for $A$. For each $T$ in $R(A)$, let $T^{\circ}$ be a weak limit point of $\left\{T x_{r}\right\}$ in $A^{* *}$. Then by a similar argument in the proof of Wong (1971), Lemma 2.1, we can show that $T^{\circ}$ is unique and $T^{\circ} a=$ $T(a)$ for all $a$ in $A$.

Conversely, let $T^{\circ} \in A^{* *}$. Since $T^{\circ} a \in A$ for all $a$ in $A$, it follows that the mapping $T: a \rightarrow T^{\circ} a$ is a right centralizer on A, clearly $\|T\| \leqq\left\|T^{\circ}\right\|$. Since for all $f$ in $A^{*}$,

$$
\left|T^{\circ}(f)\right|=\left|\lim _{\tau} f\left(T x_{\tau}\right)\right| \leqq\|f\|\|T\|,
$$

it follows that $\left\|T^{\circ}\right\| \leqq\|T\|$ and so they are equal. The lemma now follows.

For each right centralizer $T$ on $A$ and $x$ in $A_{1}$, by Wong (1974), Lemma 3.6, $T x \in A_{1}$ and $|T x|_{1} \leqq\|T\||x|_{1}$. We define

$$
F_{T}(x)=\operatorname{tr}(T x) \quad(x \in A) .
$$

Lemma 3.2. For each $T$ in $R(A), F_{T}$ is an element in $A_{1}^{*}$ with $\left\|F_{T}\right\|=$ $\|T\|$. Conversely, for each $F$ in $A_{1}^{*}$, there exists some $T$ in $R(A)$ such that $F=F_{T}$.

Proof. Let $T$ be a right centralizer on $A$. Since for all $x$ in $A_{1}$,

$$
\left|F_{T}(x)\right|=|\operatorname{tr}(T x)| \leqq|T x|_{1} \leqq\|T\||x|_{1},
$$

it follows that $F_{T} \in A_{1}^{*}$ and $\left\|F_{T}\right\| \leqq\|T\|$. We show that $\left\|F_{T}\right\| \geqq\|T\|$. In fact, let $a \in A$ and $\left\{e_{\tau}\right\}$ a maximal orthogonal family of hermitian minimal idempotents in $A$ such that $(T a)(T a)^{*} e_{\tau}=e_{\tau}(T a)(T a)^{*} e_{\tau}$. Then

$$
\begin{aligned}
\left\|e_{\tau}(T a)(T a)^{*} e_{\tau}\right\| & =\operatorname{tr}\left(e_{\tau}(T a)(T a)^{*} e_{\tau}\right) \text { (by Wong (1974), Lemma 4.4) } \\
& =\operatorname{tr}\left(T\left(a(T a)^{*} e_{\tau}\right)\right) \leqq\left\|F_{T}\right\|\left|a(T a)^{*} e_{\tau}\right|_{1} \\
& \leqq\left\|F_{T}\right\|\|a\|\|T a\|\left|e_{\tau}\right|_{1} \text { (by Wong (1974), Lemma 3.6) } \\
& =\left\|F_{T}\right\|\|a\|\|T a\|,
\end{aligned}
$$

where the last equality in (3.2) follows from the fact that $\left|e_{r}\right|_{1}=1$ (Wong 
(1974), Lemma 3.1). Since $\left\{e_{\tau}\right\}$ is maximal and commutes with $(T a)(T a)^{*}$, it follows easily that

$$
\begin{aligned}
\|T a\|^{2} & =\left\|(T a)(T a)^{*}\right\|=\sup _{+}\left\|e_{\tau}(T a)(T a)^{*} e_{r}\right\| \\
& \leqq\left\|F_{T}\right\|\|a\|\|T a\| .
\end{aligned}
$$

Therefore it follows immediately from (3.3) that $\|T\| \leqq\left\|F_{T}\right\|$ and so they are equal.

Conversely, let $F$ be an element in $A_{1}^{*}$. Then by Saworotnow (1970), Theorem 2, $F$ gives a right centralizer $S$ on $A_{2}$ such that $\operatorname{tr}(S x)=$ $F(x)\left(x \in A_{1}\right)$ and $\|S\|=\|F\|$. We show that $S$ can be extended to a right centralizer $T$ on $A$. In fact, let $a$ be an element in the socle of $A$. Then $a \in$ $A_{2}$. Let $\left\{f_{r}\right\}$ be a maximal orthogonal family of hermitian minimal idempotents in $A$ such that $(S a)(S a)^{*} f_{\tau}=f_{\tau}(S a)(S a)^{*} f_{\tau}$. By (3.2) and (3.3), we see that $\|S a\|^{2} \leqq\|F\|\|a\|\|S a\|$ and so $\|S a\| \leqq\|F\|\|a\|$. Since the socle of $A$ is dense in $A$, it follows easily that $S$ admits an extension $T$ in $R(A)$ and this completes the proof.

Now we have a generalization of Schatten (1960), Theorem 2.

THEOREM 3.3. Let $A$ be a dual $B^{*}$-algebra. Then the conjugate space of $A_{1}$ is $A^{* *}$.

Proof. This result immediately follows from Lemma 3.1 and Lemma 3.2.

In Wong (1974), p. 367, we had shown that $A^{*}=A_{1}$. Hence Schatten (1960), Theorem 3 holds for an arbitrary dual $B^{*}$-algebra.

Let $A^{\perp}$ be the subspace of $A^{* * *}$ which vanishes identically on $A \subset A^{* *}$. If $a$ is in $A_{1}$, then the expression $\operatorname{tr}(a x)(x \in A)$ gives a linear functional in $A^{*}$ (see Wong (1974)). Since $a T \in A_{1}\left(T \in A^{* *}\right)$, the expression $\operatorname{tr}(a T)$ also gives a linear functional in $A^{* * *}$. Thus we can identify $A_{1}$ as a subspace of $A^{* * *}$. If $a \in A^{\perp} \cap A_{1}$, then $\operatorname{tr}(a x)=0$ for all $x \in A$ and $\operatorname{so} \operatorname{tr}\left(a a^{*} e\right)=$ $\operatorname{tr}\left(e a a^{*} e\right)=0$ for any hermitian minimal idempotent $e \in A$. Hence $\left\|e a a^{*} e\right\|=\operatorname{tr}\left(e a a^{*} e\right)=0$ and so $e a=0$. Since $e$ is arbitrary, it follows that $a=0$. Consequently $A^{\perp} \cap A_{1}=(0)$.

The following theorem is a generalization of a result by Dixmier. The argument used here is similar to that given in the proof of Schatten (1960), Theorem 5.

THEOREM 3.4. Let $f$ be a continuous linear functional on $A^{* *}$. Then $F=G+H$ uniquely with $G \in A_{1}, H \in A^{\perp}$ and $\|F\|=\|G\|+\|H\|$.

Proof. By the proof of Schatten (1960), Theorem 5, it is sufficient to show that $\|F\| \geqq\|G\|+\|H\|$. Let $\delta>0$ be given. Write $G=W Q$, where 
$W \in R(A)$ with $\|W\|=1$ and $Q=\left(G^{*} G\right)^{1 / 2}$ see Wong (1974). By Lemma 3.1 , we can assume that $W \in A^{* *}$. Let $Q=\Sigma_{j} k_{j} e_{j}=\Sigma_{\tau} e_{\tau} Q$ be a spectral representation of $Q$ (see (2.1)). By Wong (1974), Lemma 3.1, $|Q|_{1}=|G|_{1}=\Sigma_{j} k_{j}$. Since $\left\{e_{r}\right\}$ is maximal, $\Sigma_{\tau} e_{\tau} A$ is dense in $A$ and so we can choose some $a \in$ $\Sigma_{\tau} e_{\tau} A$ with $\|a\| \leqq 1$ such that

$$
G(a)>\|G\|-\delta .
$$

Write $a=\sum_{j=1}^{m} e_{r} a_{j}$ with $a_{i} \in A$ and $e_{\tau_{j}} \in\left\{e_{r}\right\}$. Choose $n$ so large that $\Sigma_{j>n}^{j} k_{j}>$ $\delta$ and choose $b^{\circ} \in A^{* *}$ with $\left\|b^{\circ}\right\| \leqq 1$ such that

$$
H\left(b^{\circ}\right)>\|H\|-\delta \text {. }
$$

Let $E=\left\{e_{j}\right\}_{i=1}^{n} \cup\left\{e_{\tau_{i}}\right\}_{j=1}^{m}$. Then $E \subset\left\{e_{\tau}\right\}$ and $e_{\tau} E=(0)$ if $e_{\tau} \notin E$. Let $P=$ $\Sigma\{e: e \in E\}$ and $b=(1-P) b^{\circ}(1-P)$. Since $-\|1-P\|=1,\|b\| \leqq 1$. Since $a=P a$, we have

$$
\|a+b\|=\max (\|a\|,\|b\|) \leqq 1 .
$$

Also

$$
G b=W Q b=W\left(\sum_{j} k_{j} e_{j} b\right)=W\left(\sum_{j>n} k_{j} e_{j}\right) b .
$$

By identifying $A_{1}$ as a subspace of $A^{* * *}$, it follows from (3.7) that

$$
\begin{aligned}
G(b)=\operatorname{tr}(G b) \leqq|G b|_{1} & \leqq\left|\sum_{j>n} k_{j} e_{j}\right|_{1} \\
& =\sum_{j>n} k_{j}<\delta .
\end{aligned}
$$

Since $a$ and $b-b^{\circ}$ are in $A$, we have $H(a)=0$ and $H(b)=H\left(b^{\circ}\right)$. It follows now easily from (3.4), (3.5), (3.6) and (3.8) that $\|F\| \geqq\|G\|+\|H\|$ and this completes the proof.

\section{A three lines theorem for $A_{p}(1 \leqq p \leqq \infty)$}

For $1 \leqq p \leqq \infty$, it was shown in Wong (1974) that $A_{p}$ can be identified with $A_{q}^{*}\left(p^{-1}+q^{-1}=1\right)$. In fact, for each $F$ in $A_{q}^{*}$, there exists some $a$ in $A_{p}$ such that $F(x)=\operatorname{tr}(a x)\left(x \in A_{q}\right)$ and $\|F\|=|a|_{p}$.

In this section, $S_{A}$ will denote the socle of $A$. By Wong (1974), Theorem 3.9, $S_{A}$ is dense in $A_{p}(1 \leqq p \leqq \infty)$.

Let $a(\neq 0)$ be a positive element in $A$ with a spectral representation $a=$ $\Sigma_{n} k_{n} e_{n}$. Then $k_{n}>0$. Let $z$ be a complex number. Define $a^{z}(\operatorname{Re} z \geqq 0)$ by $a^{z}=\sum_{n} k_{n}^{z} e_{n}$, where $k_{n}^{z}=e^{z \ln \left(k_{n}\right)}$. If $a \in S_{A}$, then it is clear that $A^{z}$ is always well defined. 
Lemma 4.1: Let $a \in A^{* *}$ and $p^{-1}+q^{-1}=1(1 \leqq p<\infty)$. Then $a \in A_{p}$ if and only if

$$
\sup \left\{|\operatorname{tr}(a s)| /|s|_{q}: s \in S_{A}\right\}<\infty .
$$

Proof. If $a \in A_{p}$, then by Wong (1974), Lemma 4.2,

$$
|\operatorname{tr}(a s)| \leqq \mid \text { as }\left.\right|_{1} \leqq|a|_{p}|s|_{q} \quad\left(s \in S_{A}\right) .
$$

Hence (4.1) holds. Conversely, suppose (4.1) holds. Then the functional $F(s)=\operatorname{tr}(a s)$ is continuous on $S_{A}$ (with norm $|\cdot|_{q}$ ). Since $S_{A}$ is dense in $A_{q}$, it follows that $F$ can be extended to a linear functional in $A_{q}^{*}$. Hence by Wong (1974), Theorem 6.2, there exists some $b$ in $A_{p}$ such that $F(x)=\operatorname{tr}(b x)$ for all $x \in A_{q}$. Thus $\operatorname{tr}(a s)=\operatorname{tr}(b s)$ for all $s \in S_{A}$. Let $e$ be a hermitian minimal idempotent in $A$. Since $a^{*} e \in S_{\mathrm{A}}$, we have

$$
\operatorname{tr}\left(e a a^{*} e\right)=\operatorname{tr}\left(a a^{*} e\right)=\operatorname{tr}\left(b a^{*} e\right)=\operatorname{tr}\left(e b a^{*} e\right) .
$$

Since by Wong (1974), Lemma 4.4, eaa* $e=\left(\operatorname{tr}\left(e a a^{*} e\right)\right) e$ and $e b a^{*} e=$ $\left(\operatorname{tr}\left(e b a^{*} e\right)\right) e$, it follows from (4.2) that $e a a^{*} e=e a b^{*} e$. Similarly $e a b^{*} e=$ $e b b^{*} e$. Consequently, $e(a-b)(a-b)^{*} e=0$ and so $e(a-b)=0$. Since $e$ is arbitrary, we see easily that $A(a-b)=0$. Thus $A^{* *}(a-b)=0$ and so $a=$ $b \in A_{p}$. This completes the proof.

Remark. Some arguments in the above proof are similar to those in the proof of Gohberg and Krein (1969), Lemma 12.1.

Let $E_{A}$ be the set of all hermitian minimal idempotents in $A$. For each $e \in E_{A}, a \in A$ and $T \in A^{* *}$, let $k(e, T, a)$ be the constant such that $k(e, T, a) e=e T a e$.

Definition. Let $G$ be a region in the complex plane. We call $\left\{T_{z} \in\right.$ $\left.A^{* *}: z \in G\right\}$ holomorphic in the region $G$, if for any $e \in E_{A}$ and $a \in A$, the scalar function $z \rightarrow k\left(e, T_{z}, a\right)$ is holomorphic in $G$.

Remark. It is easy to see that if $A$ is a simple algebra, then the above definition is equivalent to that given in Gohberg and Krein (1969), since $A=$ $L C(H)$ for some Hilbert space $H$ and $e \in E_{\mathrm{A}}$ has the form $e=x \otimes x$ with $x$ in $H$.

Now we have the main result in this section which is a generalization of Gohberg and Krein (1969), Theorem 13.1.

Theorem 4.2. Let $\left\{T_{z} \in A^{* *}: u \leqq \operatorname{Re} z \leqq v\right\}(u<v)$ be holomorphic. Suppose that $T_{z} \in A_{r_{1}}\left(1 \leqq r_{1}<x\right)$ on the line $z=u+i y(-\infty<y<\infty)$ and $T_{z} \in A_{r_{2}}\left(r_{1}<r_{2} \leqq x\right)$ on the line $z=v+i y(-\infty<y<x)$. If $\left|T_{u+i y}\right|_{r_{1}} \leqq C_{1}$, $\left|T_{v+i y}\right|_{r_{2}} \leqq C_{2}(-x<y<\infty)$ and 


$$
\ln \mid k\left(e, T_{z}, a \mid \leqq N_{e, a} \exp \left(k_{e, a}|\operatorname{Im} z|\right),\right.
$$

where $u<\operatorname{Re} z<v, e \in E_{A}, a \in A$ and $0 \leqq k_{e, a}<\pi /(v-u)$, then, for all $z=$ $x+i y \quad(u<x<v,-x<y<x), \quad T_{z}=T_{x+i y} \in A_{r}$ and $\left|T_{x-i y}\right| . \leqq C_{1}^{1-t} x C_{z}^{t}$, where $t_{x}=(x-u) /(v-u)$ and $r^{-1}=r_{1}^{-1}+t_{x}\left(r_{2}^{-1}-r_{1}^{-1}\right)$.

Proof. Let $s \in S_{A}$ with $|s|_{r^{\prime}}=1\left(\left(r^{\prime}\right)^{-1}+r^{-1}=1\right)$ and $G=\left(s^{*} s\right)^{1 / 2}$. Then we can write $s=W G$ and $G=W^{*} s$, where $W, W^{*} \in R(A)$ (see Wong (1974)). By Lemma 3.1, we can assume that $W$ and $W^{*}$ are in $A^{* *}$. Hence $G \in S_{A}$ and so it has a spectral representation $G=\sum_{j=1}^{n} k_{j} e_{j}$. Consider the function

where

$$
f(z)=\operatorname{tr}\left(T_{a} W G^{c+d z}\right) \quad(u \leqq \operatorname{Re} z \leqq v),
$$

$$
c+d z=r^{\prime}\left((v-z) /(v-u) r_{1}^{\prime}+(z-u) /(v-u) r_{2}^{\prime}\right),
$$

$\left(r_{1}^{\prime-1}+r_{j}^{-1}=1 ; j=1,2\right)$. Then it is easy to see that $f(z)=\sum_{j-1}^{n} k_{j}$, where $k_{j}=$ $k\left(e_{i}, T_{z}, W G^{c+d z}\right)$. Hence $f(z)$ is holomorphic on $u \leqq \operatorname{Re} z \leqq v$. Now by using Lemma 4.1 and the argument in the proof of Gohberg and Krein (1969), Theorem 13.1, we can prove the theorem.

\section{References}

I. C. Gohberg and M. G. Krein (1969), 'Introduction to the theory of linear nonselfadjoint operators in a Hilbert space', (Transl. Math. Monographs, 18, Amer. Math. Soc., Providence. R. I., 1969).

C. A. McCarthy (1967), 'c $c_{p}$ ', Israel J. Math. 5, 249-271.

C. E. Rickart (1960), 'General theory of Banach algebras', (University series in Higher Math., Van Nostrand. Princeton, N. J. 1960).

S. Sakai (1971), 'C*-ałgebras and $W^{*}$-algebras', (Ergebnisse der Math. und ihrer Grenzgebiete, Heft 60, Springer-Verlag, Berlin, 1971).

P. P. Saworotnow (1970), 'Trace-class and centralizers of an $H^{*}$-algebra', Proc. Amer. Math. Soc. 26, 101-104.

P. P. Saworotnow and J. C. Friedell (1970), 'Trace-class for an arbitrary $H^{*}$-algebra', Proc. Amer. Soc. 26, 95-100.

R. Schatten (1960), 'Norm ideals of completely continuous operators', (Ergebnisse der Math. und ihrer Grenzgebiete, Heft 27, Springer-Verlag, Berlin, 1960).

P. K. Wong (1971), 'On the Arens product and annihilator algebras', Proc. Amer. Math. Soc. 30, $79-83$

P. K. Wong (1973), 'On the Arens products and certain Banach algebras', Trans. Amer. Math. Soc. $180,437-448$.

P. K. Wong (1974), 'The $p$-class in a dual $B^{*}$-algebra', Trans. Amer. Math. Soc. 200, 355-368.

Department of Mathematics.

Seton Hall University,

South Orange,

New Jersey 07079 ,

U.S.A. 